\title{
Real-World Persistence, Maintenance Dosing, and Pre-Post Corticosteroid and Opioid Use Among Crohn's Disease Patients with Prescription Claims for Ustekinumab in the USA
}

\author{
Zhijie Ding $^{1} \cdot$ Camilo Obando $^{1} \cdot$ Erik Muser $^{1} \cdot$ Chris Kozma $^{2} \cdot$ Terra Slaton $^{3}$
}

Accepted: 18 May 2021 / Published online: 16 June 2021

(c) The Author(s) 2021

\begin{abstract}
Background Real-world evidence for how US Crohn's disease (CD) patients use ustekinumab is limited.

Objectives The aim of this study was to describe the persistence, maintenance dosing, and pre-post corticosteroid and opioid use for CD patients in the USA treated with ustekinumab and those treated with adalimumab as a commonly used descriptive reference product.

Methods CD patients aged $\geq 18$ years with $\geq 2$ CD diagnoses between 1 October 2012 and 31 May 2018 and $\geq 1$ new (i.e., no claim for at least 1 year) outpatient pharmacy claim for ustekinumab or adalimumab (first claim date $=$ index date) on or after 26 September 2016 were selected from Symphony Health database. McNemar's tests were used to derive the $p$-values for pre-post changes in corticosteroid and opioid use within each treatment cohort.

Results A total of 1073 ustekinumab and 2904 adalimumab patients met analysis criteria. Using a 90-day rule for discontinuation, persistence at 1 year post-index was $69.8 \%$ for ustekinumab and $65.1 \%$ for adalimumab. The majority received doses within $\pm 30 \%$ of the approved labeling (ustekinumab $81.1 \%$; adalimumab $78.8 \%$ ). Doses higher than US package insert (PI) recommended maintenance dose were $7.0 \%$ for ustekinumab and $13.6 \%$ for adalimumab for $30 \%$ above PI, respectively; and $4.0 \%$ versus $9.4 \%$ for $50 \%$ above PI, respectively. Rates of pre-index biologic use suggest that patients treated with ustekinumab may have greater CD severity based on a greater percentage being biologic-experienced (ustekinumab 51.5\% and adalimumab $8.4 \%)$. From pre- to post-index, the relative proportion of ustekinumab patients with $\geq 1$ pharmacy claim for corticosteroids decreased by $25.5 \%(p<0.0001)$ and opioids decreased by $8.4 \%(p=0.0030)$. Results for adalimumab (a commonly used descriptive reference product in $\mathrm{CD}$ ) showed generally similar trends.

Conclusions In this real-world study, persistence for ustekinumab remained high at 1 year. The majority of the patients in the ustekinumab cohort followed US PI recommended dosing. The percentage of patients with average dose above PI recommendations over 1 year were low for ustekinumab. Reductions in the proportion of patients with claims for corticosteroids or opioids were observed in patients using ustekinumab.
\end{abstract}

Zhijie Ding

zding17@ITS.JNJ.com

Janssen Scientific Affairs, LLC, Horsham, PA, USA

2 Independent Consultant, St Helena Island, SC, USA

3 Independent Consultant, West Columbia, SC, USA 


\section{Key Points}

Approximately half of the patients with $\mathrm{CD}$ using ustekinumab had claims for other biologics in the 12 months prior to index, evidence that it was frequently being used in biologic-refractory patients (note incomplete medical claims limitation which likely means this is an underestimate of patients with prior biologic experience).

The majority of patients appeared to be persistent with ustekinumab treatment 1 year post-index.

The majority of patients with ustekinumab followed US PI recommended dosing. The percentages of patients with average dose above PI recommendations over 1 year were low for ustekinumab.

Pre- and post-treatment analysis showed lower postindex proportions of patients with claims for corticosteroids and opioids, indicating a potentially positive treatment outcome.

Key limitations of the study include the use of prescription claims data only, incompleteness of medical claims limiting availability of comorbidity data, and lack of information on disease severity.

\section{Introduction}

Crohn's Disease (CD) is a relapsing, remitting disease characterized by unpredictable, often debilitating gastrointestinal inflammatory symptoms including diarrhea, rectal bleeding, and urgency [1-3]. Lower patient quality of life is associated with disability, increased disease activity, relapses, corticosteroid treatment, psychological distress (anxiety and depression), hospitalization, and surgery [3]. Comorbidities, relapses, complications such as fistula and strictures, surgical treatment, hospitalization, and therapeutics complicate patient care and increase healthcare costs $[1,2]$. Furthermore, the incidence and prevalence of CD is increasing $[4,5]$. CD patients, especially those with colonic disease, are also at increased risk of colorectal cancer [5]. These factors create challenges for healthcare providers and payers to effectively control the disease and manage resource allocation.

Biologic treatment options are evolving and, in the USA, include adalimumab, certolizumab pegol, infliximab and biosimilars, natalizumab, ustekinumab, and vedolizumab. Anti-tumor necrosis factor- $\alpha$ (anti-TNF) products were the first biologic medications approved for moderate-to-severe CD. More recently, ustekinumab, the only biologic targeting interleukin-12 and interleukin-23, was approved by the US Food and Drug Administration (FDA) for use in moderate-to-severe CD (approved in September of 2016) [6]. Adalimumab is an anti-TNF monoclonal antibody (FDA-approved in February 2007 for use in moderate-to-severe $\mathrm{CD}$ ) that continues to be frequently used today, thus can serve as a reference product and provide descriptive context to observations among ustekinumab patients. The American College of Gastroenterology (ACG) recommends the use of adalimumab, or other anti-TNF agents, for the treatment of moderate to severe $\mathrm{CD}$ that is resistant to treatment with corticosteroids, thiopurines, or methotrexate, whereas ustekinumab should be used in patients who have failed prior treatment with corticosteroids, thiopurines, methotrexate, or anti-TNFs, or who have not had prior exposure to anti-TNFs [5].

Real-world CD treatment measures such as medication persistence, dosage escalation, and medical outcomes are highlighted as important descriptors of successful therapy in prior literature [7-9]. Medication persistence and dose escalation can be indicators of a product's efficacy and safety [10]. Persistence, as defined in most studies, refers to continuing or remaining on a CD treatment. Persistence calculations typically start at the availability of the first drug dose and end at discontinuation of therapy or upon switching to alternate therapy $[8,11]$. Persistence rates observed in $\mathrm{CD}$ patients treated with biologics were reported as $50 \%$ at 1 year [7]. Dose escalations have been reported in $8-35 \%$ of patients in the first year of therapy [9]. Factors associated with doses above package insert guidelines include primary non-response, secondary loss of response, and immunogenicity associated with loss of response [12]. Rates of dose escalation with biologics are of interest not only due to clinical implications for patients but also because of important economic implications.

In claims database studies of $\mathrm{CD}$, outcomes can be measured via changes in medical procedures (e.g., surgical interventions) before and after treatment. When medical claims are not available or extended periods of followup are not possible, change in prescription claim rates may serve as an outcome proxy. A potential successful treatment outcome might be evident from the reduction of adjunctive corticosteroid and opioid use after biologic initiation.

In light of limited real-world evidence on ustekinumab in $\mathrm{CD}$, the purpose of this study was to use prescription claims to describe the persistence, maintenance dosing, and pre-post corticosteroid and opioid use for CD patients treated with ustekinumab. To provide descriptive context to the observations for ustekinumab, claims for patients 
utilizing adalimumab were also analyzed as it is a biologic that is commonly used in CD.

\section{Methods}

\subsection{Data Source}

Data for this retrospective analysis were from a Symphony Health longitudinal database that included outpatient pharmacy, diagnosis, and procedure claims for patients with $\geq 1$ biologic indicated for any inflammatory diagnoses between 1 October 2012 through 31 May 2018 [13-16]. The Symphony Health data is collected through electronic claims processors, commercial and government (Medicare and Medicaid) sources. Symphony Health indicates that the database has representation from the US population across ages, genders, geography, and payment-type variables. The database captures more than $93 \%$ of prescriptions dispensed in the USA and territories with more than 317 million active individual patients. The database is a de-identified, HIPAA (Health Insurance Portability and Accountability Act of 1996)-compliant database. No institutional review board approval was necessary.

Obtaining resource utilization data relatively soon after approval of a medication such as ustekinumab for CD can be problematic in administrative claims data because of the lag between provision of services and payment of claims. Using comprehensive integrated claims data (i.e., insurance eligibility, prescription, outpatient and inpatient data) typically requires a wait of 12-18 months from the desired end date of an analysis due to the time lag between treatment, reporting, and the availability of research databases. Unlike most integrated medical claims databases, outpatient (i.e., retail) prescription claims databases from claims clearinghouses such as Symphony are updated daily and can be used for an early assessment of measures that can be evaluated when focusing on prescription claims and was the rationale for electing this database. Maintenance treatment with ustekinumab, like adalimumab, is administered as a subcutaneous (SC) injection and is typically reimbursed as a pharmacy benefit, meaning that claims for these biologics will appear in outpatient pharmacy claims databases.

\subsection{Study Design}

The analysis used a retrospective observational study design. Adalimumab was included to provide descriptive context since it is primarily reimbursed through pharmacy claims and is a commonly used product in CD. Given the time since initial approval for adalimumab in $\mathrm{CD}$ and the more recent approval of ustekinumab for $\mathrm{CD}$, characteristics of patients treated with each product may differ at a population level, with adalimumab used more frequently as a first line biologic than ustekinumab. Therefore, the adalimumab cohort was included to help contextualize the findings for ustekinumab.

\subsection{Observation Windows}

The identification window for study inclusion began on the Monday (26 September 2016) following the FDA approval for ustekinumab in CD (23 September 2016) and ended on 31 May 2017, which is 1 year prior to the end of the available data. The analysis index date was assigned as the date of the first claim for a maintenance dose of ustekinumab or adalimumab during this identification window. The baseline ("pre-index") period was 365 days prior to the index date. The follow-up post-observation period began on the index date and continued for 365 days.

\subsection{Analysis Index Date}

The first maintenance dose was chosen as the index event since it relies only on outpatient pharmacy claims and since the FDA-labeled induction dose of ustekinumab for $\mathrm{CD}$ requires physician office or facility-based intravenous (IV) administration with subsequent maintenance dosing delivered via subcutaneous (SC) injections. In contrast, adalimumab induction and maintenance doses are all SC. This approach was chosen due to potential limitations in completeness of medical claims in the data and differences in site-of-care for induction administration. Additionally, only non-specific medical billing codes for ustekinumab IV induction were available (the permanent J-code was issued in January 2018 after the study identification window). Thus, the first outpatient prescription claim for SC ustekinumab was assumed to be the first maintenance dose. For adalimumab, the first maintenance dose was assigned as the date of the first claim after a patient had claims for which the summed dose was above the $240 \mathrm{mg}$ threshold for induction doses.

\subsection{Patient Selection}

The primary sample included patients aged $\geq 18$ years at index with known gender, valid year of birth, and at least two Crohn's diagnoses separated by at least 30 days (ICD-9: 555.XX or ICD-10: K50.XXX) between 1 October 2012 and 31 May 2018. Patients were required to have one or more new (i.e., no study drug claim for at least 1 year) claim for ustekinumab or adalimumab on or after 26 September 2016. Because the data set does not contain information about patients' continuity in the database, proxy criteria were used 
to support an assumption of complete data. Patients were required to have one or more claim during the 365-day preindex period and one or more additional claim preceding the 365-day pre-index period. Patients were also required to have one or more pharmacy claim of any kind after index date. Patients were excluded if they had claims for the index drug billed through procedures data during the postobservation period (i.e., requirement to assume complete claims in the pharmacy data). Patients were also excluded if they had diagnoses during the 365-day pre-index period for other conditions for which the index drugs are indicated (i.e., rheumatoid arthritis, juvenile idiopathic arthritis, psoriatic arthritis, ankylosing spondylitis, ulcerative colitis, plaque psoriasis, hidradenitis suppurativa, uveitis). Patients with potential data issues (e.g., claims out of order, unclear dosages) were excluded. The index date had to be at least 365 days prior to the end of the available data (i.e., fixed 1-year observation period). The secondary maintenance dosing evaluation sample added the requirement of having at least two maintenance dosed prescriptions for inclusion.

\subsection{Variables}

\subsubsection{Demographics and Patient Characteristics}

Demographics and patient characteristics included age, sex, patient region, type of insurance coverage, and prior biologic, corticosteroid, and opioid use.

\subsubsection{Persistence}

Duration of therapy was defined as the time between the first and last prescription fill date plus the days of supply for the last prescription and was evaluated at 1 year. Persistence is presented as the percentage of patients who did not discontinue or switch off the index medication over the 12-month follow-up period. Discontinuation was defined as no index medication observed with an allowable 90-day gap after the dispensing date of the last prescription plus the days of supply. Patients who did not switch or discontinue (i.e., were persistent) over the 12-month follow-up period were assigned a duration of therapy of 365 days. This assumes that patients who have less than a 90-day gap at the end of their observation period continued therapy until the end of the 365-day observation period.

\subsubsection{Maintenance Dosing}

For patients with at least two maintenance-dosed claims, average maintenance dose was calculated as follows:
Step 1. Calculate mg per prescription as the product strength (based on NDC) multiplied by quantity dispensed in units (number of injections) for each claim.

Step 2. Calculate the total mg per patient. For each patient, sum mg per prescription across claims to obtain the total mg per patient between the index date and the last study drug prescription dispensed (not counting the mg per prescription from the last claim).

Step 3. Calculate total days per patient. For each patient, sum the total number of days between index and the date of the last claim.

Step 4. Calculate mg per day per patient as the total $\mathrm{mg}$ per patient/total days per patient.

Step 5. Convert the $\mathrm{mg}$ per day into the number of $\mathrm{mg}$ per maintenance dosing interval (i.e., 56 days for ustekinumab and 14 days for adalimumab).

Step 6. Compare the average time-adjusted dose to the expected maintenance dose from the US package insert (ustekinumab $90 \mathrm{mg}$ every 8 weeks, and adalimumab $40 \mathrm{mg}$ every other week).

Maintenance dose titration variables were calculated as the percentage of patients who were $>20 \%,>30 \%,>50 \%$, and $\geq$ $100 \%$ above the US PI recommended dosage; the percentage of patients who were $>20 \%,>30 \%$, and $>50 \%$ below the US PI recommended dosage, and the percentage of patients between the upper and lower values. The percentages above and below the US PI recommended dosages were selected to provide a sensitivity analysis for the range of potential dosing.

\subsubsection{Corticosteroid and Opioid Use}

The number and percentage of patients with one or more outpatient pharmacy claim pre-index and post-index were calculated for non-topical corticosteroids and for opioids. Corticosteroid and opioid claims were identified using National Drug Codes.

\subsection{Statistical Analysis}

Chi-square and t-tests were used to test for differences in categorical and continuous variables, respectively. Fisher's Exact tests were used for categorical variables where $n<5$. McNemar's tests were used to compare proportions of patients taking opioids or corticosteroids in the pre- and post-index periods in each treatment cohort. Categorical variables were summarized using frequencies and percentages. Continuous variables were summarized with means and standard deviations. A $p$-value $<0.05$ was considered statistically significant. All analyses were performed using SAS for Windows 9.4 (Cary, NC, USA). 


\section{Results}

\subsection{Patient Characteristics in the Baseline Period}

A total of 1073 patients with a pharmacy claim for ustekinumab and 2904 patients with a pharmacy claim for adalimumab met analysis criteria (Fig. 1).

In Table 1, the mean age for ustekinumab (42.2 years) and adalimumab (42.0 years) patients was similar $(p=0.6612)$. Ustekinumab patients were comprised of more females compared to adalimumab, $60.7 \%$ versus $56.1 \%$ ( $p=0.0096)$, respectively. The regional distributions differed for the Midwest and Northeast $(p<0.0001)$ with a greater percentage of ustekinumab patients in the Northeast (31.1\% vs. $23.3 \%)$ and a lower percentage in the Midwest (22.1\% vs. 29.3\%). The most common type of insurance at index was commercial insurance $(\sim 75 \%)$ for both. There were minor differences in percentages of Medicare and Medicaid, with ustekinumab having more Medicare patients $(12.2 \%$ vs. $8.8 \% ; p=0.0014)$ and adalimumab having more Managed Medicaid (4.7\% vs. $8.9 \%$; $p<0.0001$ ).

Prior biologic exposure was found for $51.5 \%$ of ustekinumab patients in the 365 days pre-index versus $8.4 \%$ $(p<0.0001)$ of adalimumab patients, and was assessed using both medical and pharmacy data. Among patients in the ustekinumab cohort with prior exposure to biologics, $50.1 \%$ of patients (i.e., $25.8 \%$ in the full ustekinumab cohort) had one or more claim for adalimumab during the pre-index period. Notably, office-based infusions may be under-represented in the medical claims data (i.e., estimates are conservative) since the medical data may be incomplete. Therefore, estimates of prior biologic exposure may not accurately reflect actual prior biologic experience in each cohort. However, the relative differences in prior biologic exposure suggests there are substantial differences for patients treated with ustekinumab or adalimumab.

The percentage of patients with corticosteroid and opioid claims in the pre-period differed for ustekinumab and adalimumab. A greater percentage of ustekinumab patients had claims for corticosteroids $(p<0.0001)$ and opioids $(p<0.0001)$ prior to their first ustekinumab claim compared to the adalimumab group.

\subsection{Persistence}

In the 12-month follow-up period, mean duration of therapy for ustekinumab was 296 days with $69.8 \%$ of patients remaining persistent at 1 year following index while adalimumab results were 285 days and $65.1 \%$, respectively
(Fig. 2). At 1 year, for ustekinumab $24.7 \%$ of patients discontinued and 5.5\% switched from their index treatment; for adalimumab $29.2 \%$ and $5.6 \%$ discontinued and switched, respectively (Fig. 2).

\subsection{Maintenance Dosing}

Dosing was assessed for 993 ustekinumab and 2742 adalimumab patients who had at least two maintenance dose claims. The expected maintenance dosing based on the product US PIs were: (1) ustekinumab $90 \mathrm{mg}$ (SC) every 8 weeks (56 days) and (2) adalimumab $40 \mathrm{mg}$ (SC) every other week.

The majority of ustekinumab and adalimumab patients utilized maintenance doses consistent with the PI (Figs. 3 and 4).

The percentages of ustekinumab patients with average maintenance dosing above PI were $11.7 \%$ for $20 \%$ above PI, $7.0 \%$ for $30 \%$ above PI, $4.0 \%$ for $50 \%$ above PI, and $0.6 \%$ for $100 \%$ above PI (Fig. 3). The percentages of ustekinumab patients who had average dosages that were below the PI maintenance dosing was $18.1 \%$ for $20 \%$ below PI, $11.9 \%$ for $30 \%$ below PI, and $4.4 \%$ for $50 \%$ below PI.

The percentages of adalimumab patients with average maintenance dosing above PI were $16.4 \%$ for $20 \%$ above PI, $13.6 \%$ for $30 \%$ above PI, $9.4 \%$ for $50 \%$ above PI, and $2.5 \%$ for $100 \%$ above PI (Fig. 4). The percentages of adalimumab patients had average dosages that were below the PI maintenance dosing was $13.6 \%$ for $20 \%$ below PI, $7.6 \%$ for $30 \%$ below PI, and $3.0 \%$ for $50 \%$ below PI.

\subsection{Pre-/Post-Corticosteroid and Opioid Claims Results}

As an assessment of the potential impact of the index biologic on other treatments, corticosteroid and opioid claims during the 365 days before (pre) and after (post) the index date were examined (Table 2). For ustekinumab there were statistically significant declines from pre- to post-index periods in the percentage of patients with one or more outpatient pharmacy claim for corticosteroids $(-25.5 \%$; $p<0.0001)$ and opioids $(-8.4 \% ; p=0.0030)$. For adalimumab, the percent decline of patients with one or more outpatient pharmacy claim for corticosteroids was $(-22.9 \% ; p<0.0001)$ and for opioids was $(-8.2 \% ; p<0.0001)$.

\section{Discussion}

In this study, real-world patient characteristics, persistence, maintenance dosing patterns, and pre-post use of corticosteroids or opioids were examined for patients with $\mathrm{CD}$ treated with ustekinumab or adalimumab. Though adalimumab was included in this analysis, it was intended as a descriptive 
Patients with known gender, valid year of birth and at least two Crohn's diagnoses separated by at least 30 days (ICD-9: 555.XX or ICD-10: K50.XXX) between 10/1/2012 and 5/31/2018 ustekinumab $(\mathrm{n}=532,152)$

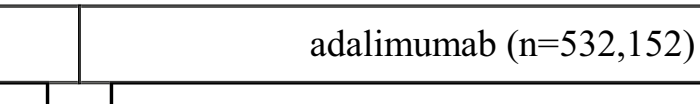

Had at least 1 new (i.e., no claim for at least 1-year) claim for ustekinumab or adalimumab on or after $9 / 26 / 2016$

\begin{tabular}{|l|l|}
\hline ustekinumab $(\mathrm{n}=6,430)$ & adalimumab $(\mathrm{n}=20,692)$ \\
\hline ustekinumab $(\mathrm{n}=6,260)$ & Age $\geq 18$ years at index \\
\hline
\end{tabular}

Met proxy for complete claims in pharmacy data and medical data: $\geq 1$ claim more than 365 days prior to the index date and $\geq 1$ claim during the 365 days prior to the index date

ustekinumab $(n=4,978)$

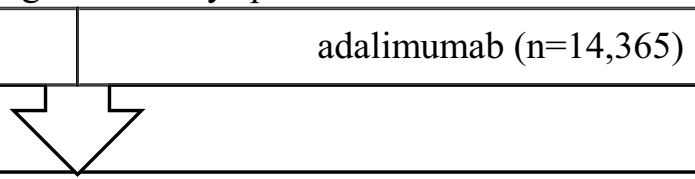

Met proxy for post-index claim activity: $\geq 1$ pharmacy claim of any kind after index date

ustekinumab $(n=4,786)$

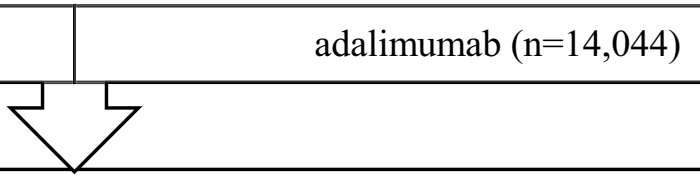

Excluded patients with claims for index drug billed through procedures data during the post observation period (i.e., requirement to assume complete claims in the pharmacy data)

ustekinumab $(\mathrm{n}=4,528)$

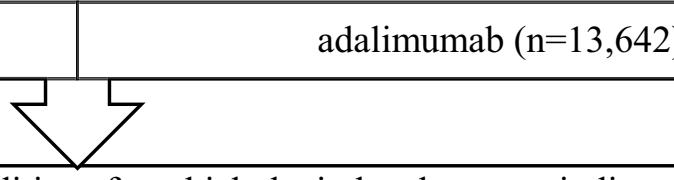

Excluded patients with diagnoses for other conditions for which the index drugs are indicated; during the 365-day pre-index period

ustekinumab $(\mathrm{n}=3,735)$

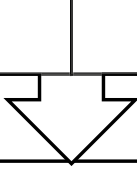

adalimumab $(\mathrm{n}=11,159)$

Retain patients that have at least one maintenance dose claim and no potential data issues ustekinumab $(\mathrm{n}=3,519)$

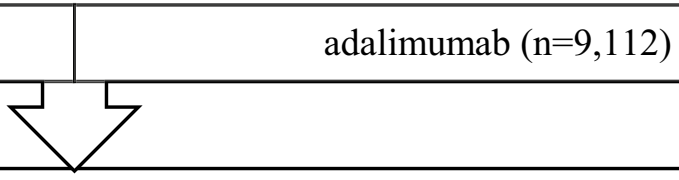

Retain patients with an index date at least 365-days before the end of the available data 
Table 1 Ustekinumab and adalimumab patient characteristics (baseline 12 months pre-index)

\begin{tabular}{llll}
\hline Characteristic & $\begin{array}{l}\text { Usteki- } \\
\text { numab }(n= \\
1073)\end{array}$ & $\begin{array}{l}\text { Adali- } \\
\text { mumab }(n \\
=2904)\end{array}$ & \\
\hline Age at index, years, mean & & \\
$\quad$ (SD) & $42.2(14.4)$ & $42.0(14.7)$ & 0.6612 \\
Female, $n(\%)$ & $651(60.7)$ & $1629(56.1)$ & 0.0096 \\
Region, $n(\%)$ & & & \\
Midwest & $237(22.1)$ & $850(29.3)$ & $<0.0001$ \\
Northeast & $334(31.1)$ & $677(23.3)$ & $<0.0001$ \\
South & $357(33.3)$ & $1039(35.8)$ & 0.1415 \\
West & $144(13.4)$ & $337(11.6)$ & 0.1191 \\
Unknown & $1(0.1)$ & $1(0.0)$ & 0.4669 \\
Payment type-index claim & & & \\
Commercial & $818(76.2)$ & $2155(74.2)$ & 0.1916 \\
Medicare & $131(12.2)$ & $256(8.8)$ & 0.0014 \\
Assistance Programs & $53(4.9)$ & $146(5.0)$ & 0.9099 \\
Managed Medicaid & $50(4.7)$ & $259(8.9)$ & $<0.0001$ \\
Medicaid & $20(1.9)$ & $85(2.9)$ & 0.0635 \\
Cash & $1(0.1)$ & $3(0.1)$ & 1.0000 \\
Prior biologic & $553(51.5)$ & $245(8.4)$ & $<0.0001$ \\
Prior treatment & & & $<0.0001$ \\
Corticosteroids & $764(71.2)$ & $1781(61.3)$ & $<0001$ \\
Opioids & $655(61.0)$ & $1445(49.8)$ & $<0.0001$ \\
\hline
\end{tabular}

${ }^{a}$ Pairwise comparisons using Chi-square where $\mathrm{n} \geq 5$ and Fisher's Exact where $n<5$

Unadjusted $p$-values are shown reference product. Direct comparison of follow-up outcomes for the two treatments was not performed given expected differences in the patient populations using these products and limited ability to control for baseline differences in each cohort using this data source. In addition, given its more recent approval for $\mathrm{CD}$, it is likely that more of the patients treated with ustekinumab in our study were refractory patients having failed prior biologics and perhaps with more severe disease on average than the adalimumab patients. Evidence of these differences in the patient populations were observed in the proportion of pre-index biologic use (ustekinumab 51.5\% and adalimumab $8.4 \%$ ) and preindex corticosteroid and opioid claims, with a greater proportion of ustekinumab patients having pre-index claims for corticosteroids and opioids relative to adalimumab. When interpreting treatment results, it is important to consider that baseline patient characteristics differ for the two cohorts in this descriptive study (i.e., gender, region, payer, and prior biologic and corticosteroid and opioid claims).

This study found that the percentage of patients who were persistent with the index therapy at 1 year after the index date was $69.8 \%$ with ustekinumab and $65.1 \%$ with adalimumab. Notably, the ustekinumab results are lower than results reported previously from a study using a potentially more complete claims data set that found ustekinumab persistence at 1 year of $83.6 \%$ [8]. In contrast, persistence observed with adalimumab in our study was somewhat higher than what has been reported in some other studies. For example, Chen et al. examined treatment with infliximab, adalimumab, certolizumab pegol, golimumab, and vedolizumab, and observed that half of all patients on biologics discontinued treatment within 1 year [7]. Govani

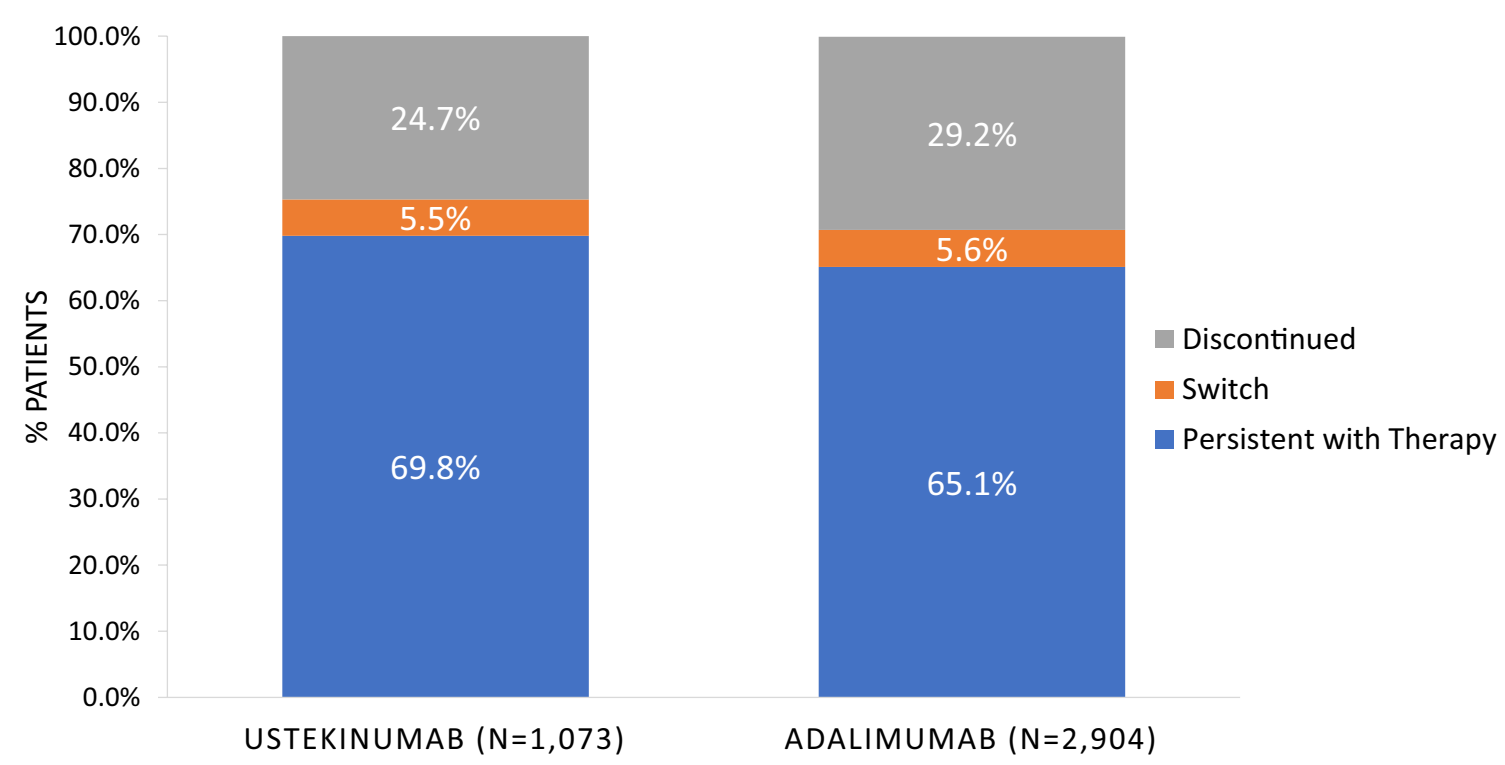

Fig. 2 Percentage of patients at 1 year who are persistent with therapy, switch or discontinue, by study drug 


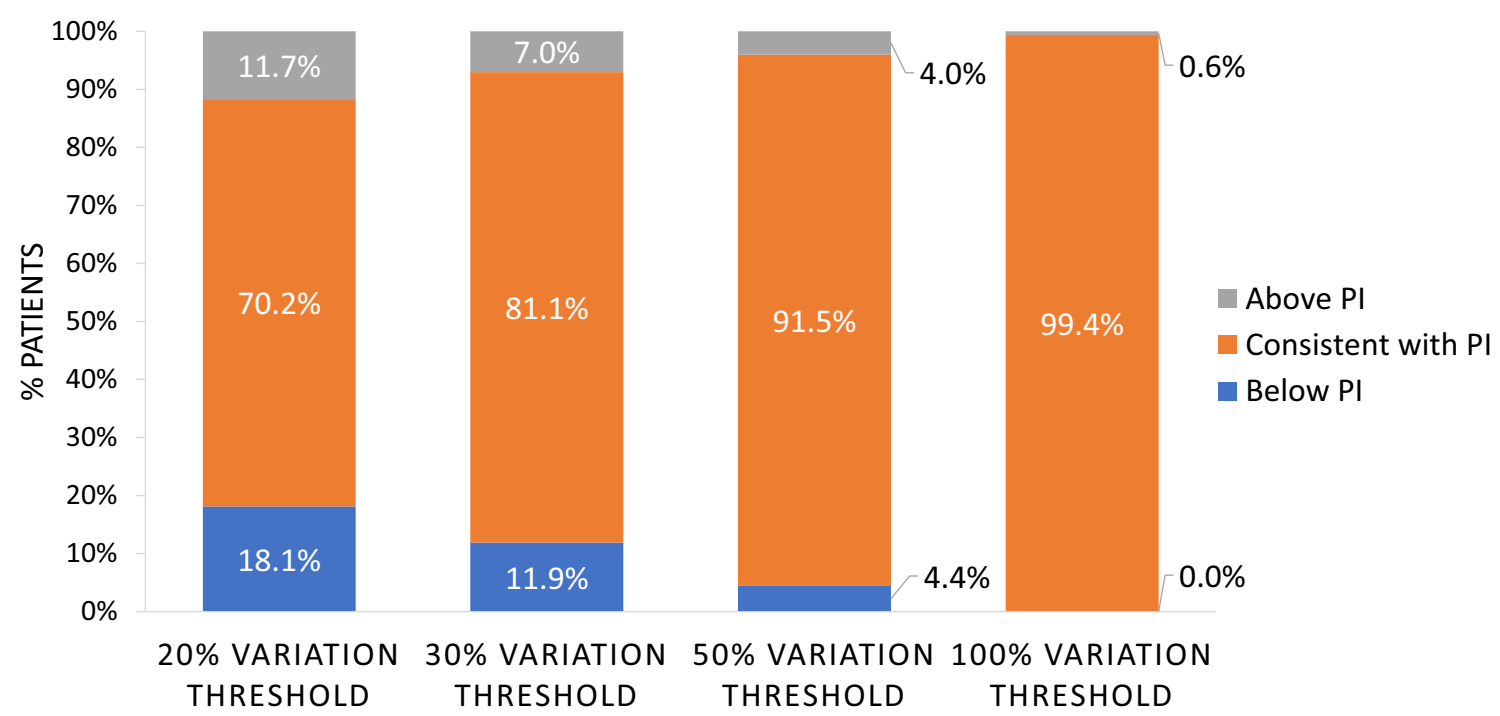

Fig. 3 Percent of ustekinumab patients by dosing that is above, consistent with, and below US package insert (PI) recommendations by $20 \%$, $30 \%, 50 \%$, or $100 \%$

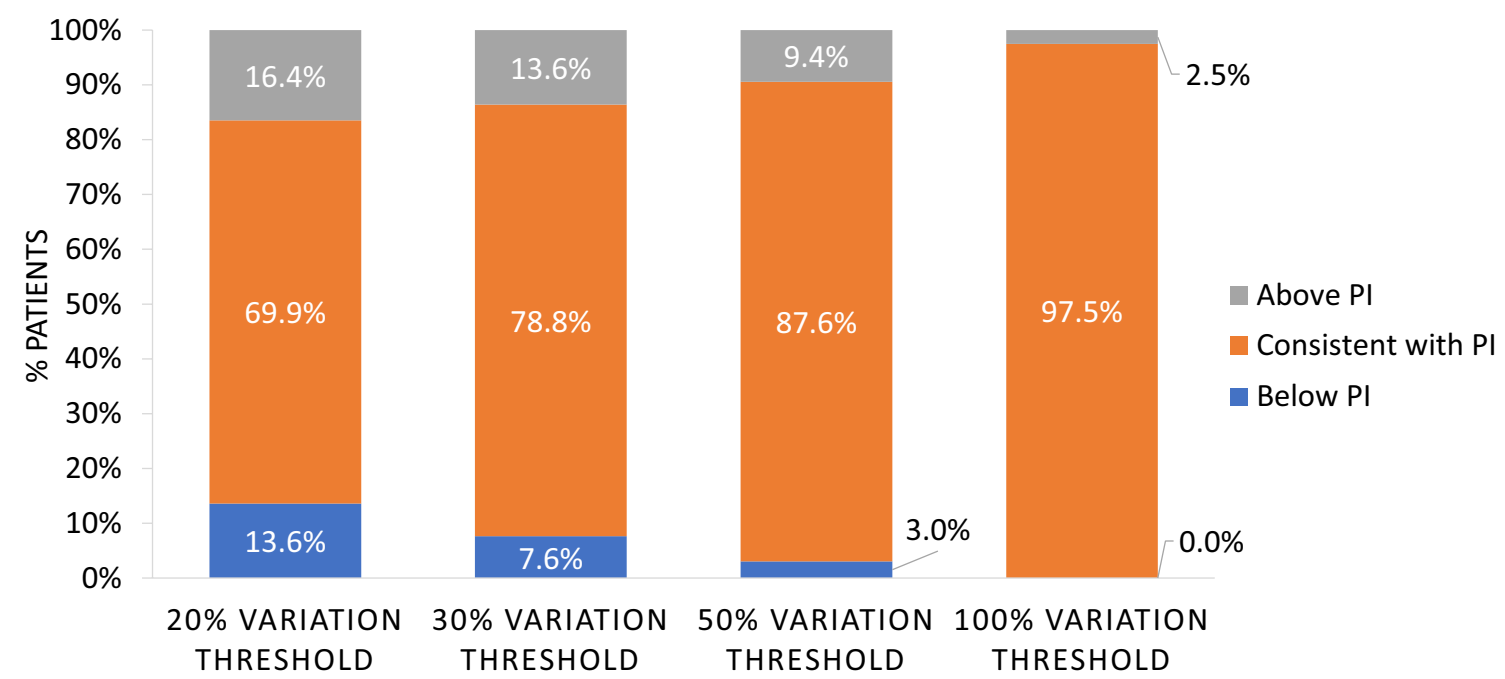

Fig. 4 Percent of adalimumab patients by dosing that is above, consistent with, and below US package insert (PI) recommendations by $20 \%$, $30 \%, 50 \%$, or $100 \%$

Table 2 Number and percentage of patients with prescription claims treatment for corticosteroids and opioids during the 365 days before and after index

\begin{tabular}{|c|c|c|c|c|c|c|c|c|}
\hline \multirow[t]{2}{*}{ Drug category $^{b}$} & \multicolumn{4}{|c|}{ Ustekinumab $(n=1073)$} & \multicolumn{4}{|c|}{ Adalimumab $(n=2904)$} \\
\hline & $\begin{array}{l}\text { Pre-index } \\
n\left(\%^{\mathrm{a}}\right)\end{array}$ & $\begin{array}{l}\text { Post-index } \\
n\left(\%^{\mathrm{a}}\right)\end{array}$ & $\%$ Change & $p$-values ${ }^{\mathrm{a}}$ & $\begin{array}{l}\text { Pre-index } \\
n\left(\%^{\mathrm{a}}\right)\end{array}$ & $\begin{array}{l}\text { Post-index } \\
n\left(\%^{\mathrm{a}}\right)\end{array}$ & $\%$ Change & $p$-values ${ }^{\mathrm{a}}$ \\
\hline Corticosteroids & $764(71.2)$ & $569(53.0)$ & $-25.5 \%$ & $<0.0001$ & $1781(61.3)$ & $1374(47.3)$ & $-22.9 \%$ & $<0.0001$ \\
\hline Opioids & $655(61.0)$ & $600(55.9)$ & $-8.4 \%$ & 0.0030 & $1445(49.8)$ & $1326(45.7)$ & $-8.2 \%$ & $<0.0001$ \\
\hline
\end{tabular}

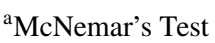

${ }^{b}$ Patients can be in multiple rows; denominators are the number of patients in each study group 
et al. reported persistence of $55 \%$ for a privately insured population while persistence was $75 \%$ for a Veteran's Health Administration cohort in the 12-month follow-up after patients initiated adalimumab [17]. The difference in persistence reported in our study may be explained by slightly different methodology (i.e., focus on maintenance therapy), different databases, and a much wider patient identification window (reflecting potential underlying change in treatment options and guidelines over time). Disease progression, loss of response, infection, treatment side effects, and disease progression or symptom worsening are factors that may lead to treatment discontinuation [7]. Additional studies using a closed (or more complete) data source with a larger sample size, and a longer follow-up period or comparative effectiveness approach controlling for differences in baseline characteristics and confounders are needed to further evaluate how effective these two medications are in the real world.

Maintenance dosing was assessed for the subgroups with at least two maintenance dose claims for the index biologics. Overall, the majority of ustekinumab and adalimumab patients received doses consistent with approved labeling in the USA. A small percentage of patients were above and below the US PI recommended doses. Upward dose titration may be associated with loss of response, and has been observed in $28 \%$ of CD patients initiating biologic treatment [18]. One study reported ustekinumab dose escalation (with a $20 \%$ variation threshold definition) was observed in $17.9 \%$ of $\mathrm{CD}$ patients [8]. Dose escalation for adalimumab has been observed in $13-34 \%$ of patients at 7 months after treatment initiation $[18,19]$. The differences we observed between our results and others might be due to a different study sample, different methodologies used, or different datasets. Future studies are warranted to assess the maintenance dosing for these medications.

In the absence of complete medical claims information, prescription medication claims can be used as a proxy indicator for disease and symptom control in CD. In our study, reductions in the proportion of patients with corticosteroid and opioid prescriptions pre- to post-biologic use were observed for both ustekinumab and adalimumab. The reduction in the percent of patients with claims for corticosteroids and opioids in the post-treatment period may serve as a surrogate measure of the positive impact on patient outcomes (or at least the reduced need for additional medications to manage their disease or resulting symptoms). While this real-world data was limited to prescription claims, further research using medical claims would add beneficial information about outcomes such as office visits, ED visits, and surgical procedures. Since medical claims data were incomplete in this data source, we did not examine such outcomes. Still, studies using prescription claims data are a reasonable way to assess the real-world effectiveness of treatment with biologics. Pre- and post-treatment analysis showed significantly lower post-index proportions of ustekinumab patients with claims for corticosteroids and opioids in this mostly bioexperienced population, indicating an ability to help address ongoing corticosteroid and opioid use as an unmet need in CD patients.

The proportion of patients with prescriptions for opioids in the present study is comparable to that reported in other recent studies. In a retrospective study of patients with CD using the OptumHealth Care Solutions database, $72 \%$ of patients had a claim for opioids over a mean observation period of 56.6 months [20]. In another study of patients with $\mathrm{CD}$ that used pooled data from three large national, commercial databases, $54 \%$ of patients had at least one opioid claim in the 12-month baseline period in the USA [8]. These rates of opioid use are generally consistent with those reported in the present study, which ranged from $45.7 \%$ (adalimumab cohort in the post-index period) to $61.0 \%$ (ustekinumab cohort in the pre-index period).

Our study has several limitations. As noted previously, prescription claims data, while timely, offers only a limited set of medical parameters. Further, errors in coding may impact our results. The index date in our study was the first outpatient prescription claim after the total induction dose had been dispensed for adalimumab. However, patients might have prescriptions dispensed or administered at facilities that do not contribute data to the Symphony Health Solutions database. Ustekinumab IV induction doses are typically administered in facilities where claims data may be less complete and definitive identification may not be possible since the permanent medical billing code ( $\mathrm{j}$-code) was not assigned until January 2018. The dosing analysis only considered the percentage of patients who met various maintenance dosing levels in the 1-year period post-index. This analysis does not account for dosage trends over time. In addition, data regarding disease severity and comorbidities were not included in the present analysis due to the incompleteness of medical claims, and claims for corticosteroids and opioids identified in this database could have been used by patients for the treatment of conditions other than $\mathrm{CD}$. Furthermore, information regarding changes in the dose of corticosteroids between the pre- and post-index periods was not included in this analysis; this is an important consideration for future analyses. Since medical data may be incomplete, estimates of prior biologic exposure may not be comprehensively reflected, particularly for products administered by IV infusion. This is also the case for patients who may have switched from the index therapy to another infusion product in the post-index period. Adalimumab was available prior to the FDA approval of ustekinumab, so prescribing patterns for a new versus older product could differ. The data do not include eligibility information (as is common with payer claims data sources); therefore, proxies of ongoing claim activity were used to support an assumption 
of complete data. The presence of a filled prescription claim and the date of a claim do not suggest whether and when the prescribed medication was actually administered. Outcomes evaluated in this analysis were observed over a brief time due to the availability of data relative to the FDA approval date for ustekinumab in CD. Evaluation over longer time periods is warranted when data are available to assess the stability and magnitude of the effect.

\section{Conclusions}

Rates of pre-period drug use suggest that $\mathrm{CD}$ patients treated with ustekinumab were mostly bio-experienced. This analysis of real-world data showed that the majority of the patients were persistent with ustekinumab and received maintenance doses consistent with approved labeling. Pre- and posttreatment analysis showed lower post-index proportions of ustekinumab patients with claims for corticosteroids and opioids, indicating a potential positive treatment outcome. While these results help quantify some of the real-world benefit of ustekinumab in CD patients, controlling for baseline patient differences and potential confounders in order to understand the relative differences between ustekinumab and other products would be a useful next step.

Acknowledgements Lavonda Miley, $\mathrm{PhD}$ participated in the writing of the manuscript.

\section{Declarations}

Funding This study was funded by Janssen Scientific Affairs, LLC.

Conflicts of interest/Competing interests $\mathrm{ZD}, \mathrm{EM}$, and $\mathrm{CO}$ are employees of Janssen Scientific Affairs, LLC (a Johnson \& Johnson company) and are Johnson \& Johnson stockholders. CK and TS received funding from Janssen Scientific Affairs, LLC (a Johnson \& Johnson company) to conduct the analyses.

Ethics approval The database is a de-identified, HIPAA (Health Insurance Portability and Accountability Act of 1996) compliant database. No institutional review board approval was necessary.

Consent to participate Data were extracted from a de-identified claims database; therefore, consent to participate was not required.

Consent for publication All authors consent to the publication of this article.

Availability of data and material Access to the database can be purchased through https://symphonyhealth.prahs.com/.

Code availability Not applicable.

Author contributions ZD, CO, EM, CK, and TS contributed to study conception and design, collection and assembly of data, and data analysis and interpretation. All authors reviewed and approved the final content of the manuscript and take full responsibility for the integrity of the work as a whole.

Previous presentation Part of the material in this article was presented at Nexus Poster Presentations at AMCP 2018 and AGA Poster Presentations at DDW® 2019

Open Access This article is licensed under a Creative Commons Attribution-NonCommercial 4.0 International License, which permits any non-commercial use, sharing, adaptation, distribution and reproduction in any medium or format, as long as you give appropriate credit to the original author(s) and the source, provide a link to the Creative Commons licence, and indicate if changes were made. The images or other third party material in this article are included in the article's Creative Commons licence, unless indicated otherwise in a credit line to the material. If material is not included in the article's Creative Commons licence and your intended use is not permitted by statutory regulation or exceeds the permitted use, you will need to obtain permission directly from the copyright holder. To view a copy of this licence, visit http://creativecommons.org/licenses/by-nc/4.0/.

\section{References}

1. Crohn's \& Colitis Foundation of America. The Facts about Inflammatory Bowel Diseases. 2019. Available from: https://www.crohn scolitisfoundation.org/sites/default/files/2019-02/Updated\% 20IBD\%20Factbook.pdf. Accessed 27 Apr 2020.

2. Rao BB, Click BH, Koutroubakis IE, et al. The cost of Crohn's disease: varied health care expenditure patterns across distinct disease trajectories. Inflamm Bowel Dis. 2017;23(1):107-15.

3. van der Have M, van der Aalst KS, Kaptein AA, et al. Determinants of health-related quality of life in Crohn's disease: a systematic review and meta-analysis. J Crohns Colitis. 2014;8(2):93-106.

4. Kappelman MD, Moore KR, Allen JK, Cook SF. Recent trends in the prevalence of Crohn's disease and ulcerative colitis in a commercially insured US population. Dig Dis Sci. 2013;58(2):519-25.

5. Lichtenstein GR, Loftus EV, Isaacs KL, Regueiro MD, Gerson LB, Sands BE. ACG clinical guideline: management of Crohn's disease in adults. Am J Gastroenterol. 2018;113(4):481-517.

6. STELARA (ustekinumab) [package insert]. Titusville, NJ: Janssen Biotech, Inc; 2016.

7. Chen C, Hartzema AG, Xiao H, et al. Real-world pattern of biologic use in patients with inflammatory bowel disease: treatment persistence, switching, and importance of concurrent immunosuppressive therapy. Inflamm Bowel Dis. 2019;25(8):1417-27.

8. Obando C, Ding Z, Muser E, et al. Persistence, dose titration, and health care resource utilization among Crohn's disease patients treated with ustekinumab: a real-world analysis in the United States. Adv Ther. 2020;37(5):2127-43.

9. Khan S, Rupniewska E, Neighbors M, Singer D, Chiarappa J, Obando C. Real-world evidence on adherence, persistence, switching and dose escalation with biologics in adult inflammatory bowel disease in the United States: a systematic review. J Clin Pharm Ther. 2019;44(4):495-507.

10. Patel H, Lissoos T, Rubin DT. Indicators of suboptimal biologic therapy over time in patients with ulcerative colitis and Crohn's disease in the United States. PLoS ONE. 2017;12(4):e0175099.

11. Cramer JA, Roy A, Burrell A, et al. Medication compliance and persistence: terminology and definitions. Value Health. 2008;11(1):44-7. 
12. Roda G, Jharap B, Neeraj N, Colombel JF. Loss of response to anti-TNFs: definition, epidemiology, and management. Clin Transl Gastroenterol. 2016;7:e135.

13. Symphony Health. Symphony Health Fact Sheet. 2020. https:// s3.us-east-1.amazonaws.com/prahs-symphony-health/resources/ Symphony_IDV-Sheet_FINAL_JULY.pdf?mtime $=2020070213$ 2440\&focal=none. Accessed 30 Apr 2021.

14. Null K, Kumar V, Lissoos T, Luo M. Infusion administration billing for vedolizumab and infliximab in inflammatory bowel disease. J Med Econ. 2018;21(11):1102-9.

15. Obando C, Woodruff K, Muser E, et al. P313 Steroid and opioid use among Crohn's patients before and after initiation of ustekinumab. J Crohns Colitis. 2018;12(supplement_1):S259-s.

16. Ding B, Kallenbach L, Slipski L, Wilk A, O’Brien D, Guranlioglu D. Patient characteristics and healthcare resource utilization among patients with COPD new to LAMA/LABA fixed-dose combination treatment in US-based real-world practice. Int $\mathrm{J}$ Chron Obstruct Pulmon Dis. 2020;15:775-86.

17. Govani SM, Lipson R, Noureldin M, et al. Adalimumab persistence for inflammatory bowel disease in veteran and insured cohorts. Am J Manag Care. 2018;24(12):e374-9.

18. Rubin DT, Mody R, Davis KL, Wang CC. Real-world assessment of therapy changes, suboptimal treatment and associated costs in patients with ulcerative colitis or Crohn's disease. Aliment Pharmacol Ther. 2014;39(10):1143-55.

19. Baert F, Glorieus E, Reenaers C, et al. Adalimumab dose escalation and dose de-escalation success rate and predictors in a large national cohort of Crohn's patients. J Crohns Colitis. 2013;7(2):154-60.

20. Manceur AM, Ding Z, Muser E, et al. Burden of Crohn's disease in the United States: long-term healthcare and work-loss related costs. J Med Econ. 2020;23(10):1092-101. 